VOL. $26(1982), 17-27$.

\title{
BOUNDARY BEHAVIOUR OF SOLUTIONS OF THE NON-PARAMETRIC LEAST AREA PROBLEM
}

\author{
LEON SIMON
}

\begin{abstract}
Previous work concerning boundary regularity of solutions of the non-parametric least area problem leaves open the question of regularity of solutions at points where the mean curvature of the boundary of the domain vanishes. We here prove that the solutions may be discontinuous at such points, even when the given boundary data is smooth. We also give a sufficient condition which will ensure continuity at such points.
\end{abstract}

Suppose $\Sigma$ is an open portion of the boundary $\partial \Omega$ of a $c^{2}$ domain $\Omega \subset \mathbf{R}^{n}$, and let $H_{\Sigma}$ be the mean curvature of $\Sigma$ relative to the inward pointing unit normal.

It is known ([1,2], [4]) that solutions of the non-parametric least area problem

$$
\int_{\Omega} \sqrt{1+|D u|^{2}}+\int_{\partial \Omega}|u-\psi| d H^{n-1} \rightarrow \min , u \in B V(\Omega),
$$

where $\psi$ is a given Lipschitz function on $\partial \Omega$, are $c^{2}(\Omega) \cap W^{1,1}(\Omega)$ functions which attain the given boundary data $\psi$ on $\Sigma$ provided $H_{\Sigma} \geq 0$. It is also known ([4]) that if $\Sigma$ is $c^{4}$ and $H_{\Sigma}<0$ on $\Sigma$, then the trace of $u$ on $\Sigma$ is a locally Lipschitz function on $\Sigma$. In either of these two cases $\left(H_{\Sigma} \geq 0\right.$ on $\Sigma, H_{\Sigma}<0$ on $\left.\Sigma\right)$ it is known

Received 15 February, 1982. 
([1], $[4,5]$ ) that $u$ extends to be Hölder continuous on $\Omega \cup \Sigma$.

These results leave open the case when $H_{\Sigma}$ changes sign on $\Sigma$ and the case when $H_{\Sigma} \leq 0$ on $\Sigma$ with $H_{\Sigma}=0$ at some point of $\Sigma$. We want to discuss these cases here. It is shown (Theorem 2) that, for the case $H_{\Sigma} \leq 0, u$ may have quite pathological trace on $\Sigma$ and may exhibit discontinuities, even in case $\Sigma$ is $C^{4}$ and $\psi$ is constant on $\Sigma$. On the other hand in case $H_{\Sigma}$ changes sign at a point $\xi \in \Sigma$ where $\partial_{\Sigma} H_{\Sigma} \neq 0$ $\left(\partial_{\Sigma}\right.$ equals tangential gradient operator on $\left.\Sigma\right), u$ must be continuous at $\xi$. In fact, at such points $\xi$, it is shown (in Theorem 1 ) that the trace of $u$ on $\Sigma$ satisfies a Lipschitz condition, and $u$ itself satisfies a Hölder condition.

Both the above theorems depend on construction of barriers, using a somewhat non-standard method of construction. For convenience the constructions here are carried out only in case $n=2$, but the reader will see that only purely technical modifications are needed to give analogous results for arbitrary $n$.

\section{Barriers}

As mentioned above, we here assume $n=2$, so that $\Sigma$ is a $C^{2}$ arc $\subset \mathbf{R}^{2}$ and $H_{\Sigma}$ denotes the curvature of $\Sigma$ relative to the inward unit normal.

The existence of boundary barriers for solutions of the minimal surface equation (and solutions of the non-parametric least area problem) in case $H_{\Sigma} \geq 0$ on $\Sigma$ has been discussed by many authors. (See for example [3], [6], [1].) Here we take a slightly different approach to the question, and obtain some new results.

To begin, we introduce convenient coordinate axes for $R^{3}$. We suppose $0 \in \Sigma$ and that $(0,1)$ is the inward pointing unit normal to $\Sigma$ at 0 , and we introduce new coordinates $\left(y_{1}, y_{2}, y_{3}\right)$ for $\mathbf{R}^{3}$ with $y_{1}=x_{1}, y_{2}=x_{3}$, and $y_{3}=x_{2}$. Then since $\Sigma$ is $c^{2}$ we have $\delta>0$ 
so that the boundary cylinder $\left(\Sigma \cap\left\{x:\left|x_{1}\right|<\delta\right\}\right) \times \mathbb{R}$ can be expressed as $\left\{\left(y_{1}, y_{2}, y_{3}\right) \in \mathbb{R}^{3}: y_{3}=\omega\left(y_{1}, y_{2}\right),\left|y_{1}\right|<\delta\right\}$, where $\omega$ is a $c^{2}$ function on $(-\delta, \delta) \times \mathbb{R}$ with $\partial \omega / \partial y_{2} \equiv 0$ and $\left(\partial \omega / \partial y_{1}\right)(0)=0$, and where

$$
\left|D \omega\left(y_{1}, y_{2}\right)\right| \leq L\left|y_{1}\right| \text { for }\left|y_{1}\right|<\delta
$$

$H_{\Sigma}$, when considered as a function on $\left(\sum \cap\left\{x:\left|x_{1}\right|<\delta\right\}\right) \times R$ which is constant on vertical lines, is given by

$$
\begin{aligned}
H_{\Sigma}\left(x_{1}, x_{2}, x_{3}\right) & \equiv H_{\Sigma}\left(y_{1}, y_{2}, y_{3}\right) \\
& =-M(\omega)\left(y_{1}, y_{2}\right) .
\end{aligned}
$$

Here $M$ is the minimal surface operator defined by

$$
M(w)=\sum_{i=1}^{2} D_{i}\left(D_{i} w / \sqrt{1+|D w|^{2}}\right)
$$

(Notice that since $\partial \omega / \partial y_{2} \equiv 0$, we can compute

$$
\left.M(\omega)=\left(\frac{\partial^{2} \omega}{\partial y_{1}^{2}}\right) /\left(1+\left(\frac{\partial \omega}{\partial y_{1}}\right)^{2}\right)^{3 / 2} .\right)
$$

With regard to the operator $M$, we need the following technical lemma, the proof of which is a simple algebraic computation. In this lemma we let the operator $M_{0}$ be given by

$$
M_{0}(w)=\left(1+|D w|^{2}\right)^{\frac{1}{2}} M(w)=\Delta w-\sum_{i, j=1}^{2} \frac{D_{i} w D_{j} w}{1+|D w|^{2}} D_{i} D_{j} w .
$$

LEMMA 1. Let $M_{0}$ be the operator defined above and let $\zeta_{1}, \zeta_{2}$ be $c^{2}$ with $\left|D \zeta_{1}\right|,\left|D \zeta_{2}\right| \leq 1$. Then

$$
\left|M_{0}\left(\zeta_{1}+\zeta_{2}\right)-M_{0}\left(\zeta_{2}\right)-\Delta \zeta_{1}\right| \leq c\left(\left|D \zeta_{1}\right|\left|D^{2} \zeta_{2}\right|+\left|D^{2} \zeta_{1}\right|\right)\left(\left|D \zeta_{1}\right|+\left|D \zeta_{2}\right|\right)
$$

where $c$ is an absolute constant.

We are going to use this lemma to construct functions $\tilde{w}$ on conical domains in the $\left(y_{1}, y_{2}\right)$ variables, such that $M(\tilde{w}) \geq 0$ and such that the 
graph $y_{3}=\tilde{w}\left(y_{1}, y_{2}\right)$ transforms to a graph $x_{3}=w\left(x_{1}, x_{2}\right)$ (under the transformation $\left.y_{1}=x_{1}, y_{2}=x_{3}, y_{3}=x_{2}\right)$. Notice that then we have $M(w)=-M(\tilde{w}) \leq 0$. In this way we construct solutions of $M(w) \leq 0$; the reason for the indirect approach (via the coordinates $y_{1}, y_{2}, y_{3}$ ) is that the computations in the $y$ coordinates are simpler, especially in view of the form we choose below for the function $\tilde{w}$.

We in fact look for a function $\tilde{w}$ of the form

$$
\tilde{w}\left(y_{1}, y_{2}\right)=\omega\left(y_{1}, y_{2}\right)+\alpha y_{2}^{k+2} \phi\left(y_{1} / y_{2}\right),
$$

where $k, \alpha$ (constants) and $\phi$ are to be chosen. We first note that, writing $f\left(y_{1}, y_{2}\right)=y_{2}^{k+2} \phi\left(y_{1} / y_{2}\right)$, we have

$$
\begin{aligned}
\frac{\partial f}{\partial y_{2}}(y) & =(k+2) y_{2}^{k+1} \phi(t)-y_{2}^{k+1} t \phi^{\prime}(t), \quad t=y_{1} / y_{2} \\
\frac{\partial^{2} f}{\partial y_{2}^{2}}(y) & =y_{2}^{k}\left[(k+2)(k+1) \phi(t)-(2 k+2) t \phi^{\prime}(t)+t^{2} \phi^{\prime \prime}(t)\right], \\
\frac{\partial^{2} f}{\partial y_{1} \partial y_{2}}(y) & =y_{2}^{k}\left[(k+1) \phi^{\prime}(t)-t \phi^{\prime \prime}(t)\right], \\
\frac{\partial f}{\partial y_{1}}(y) & =y_{2}^{k+1} \phi^{\prime}(t), \\
\frac{\partial^{2} f}{\partial y_{1}^{2}}(y) & =y_{2}^{k} \phi^{\prime \prime}(t) .
\end{aligned}
$$

Thus in particular we deduce that

$$
\Delta f(y)=y_{2}^{k}\left[\left(1+t^{2}\right) \phi^{\prime \prime}(t)-2(k+1) t \phi^{\prime}(t)+(k+2)(k+1) \phi(t)\right] .
$$

Combining these calculations, and using Lemma 1 , we deduce (taking $\tilde{w}$ as in (1.3)) that, for $K|t| \leq 1$,

$$
\begin{aligned}
& \text { (1.4) }\left|M_{0}(\tilde{w})-\left\{M_{0}(\omega)+\alpha y_{2}^{k}\left|\left(1+t^{2}\right) \phi^{\prime \prime}(t)-2(k+1) t \phi^{\prime}(t)+(k+2)(k+1) \phi(t)\right|\right\}\right| \\
& \leq \operatorname{Coy}_{2}^{k}\left(y_{2}\left(|\phi(t)|+\left|\phi^{\prime}(t)\right|\right)+\left|\phi^{\prime \prime}(t)\right|\right)\left(\left|y_{1}\right|+\alpha y_{2}^{k+1}\left(|\phi(t)|+\left|\phi^{\prime}(t)\right|\right)\right\},
\end{aligned}
$$


with $C \geq 1$ depending only on $k, \Sigma$, provided $\alpha|D f| \leq 1$.

We now assume that

(1.5) $M_{0}(\omega) \geq \mu y_{1}+R\left(y_{1}\right),\left|R\left(y_{1}\right)\right| \leq L y_{1}^{2} \leq \frac{\mu}{2}\left|y_{1}\right|,\left|y_{1}\right|<\delta$,

for some positive constants $\mu, L, \delta, L, \delta$ will (without loss of generality) be taken to coincide with the constants $L, \delta$ of (1.1).

Under the assumption (1.5), we want to discuss the possibility of selecting $\phi$ so that $(1.4)$ implies $M(\tilde{w}) \geq 0$. To do this, we take $k=1$ in the above discussion, we let $\varepsilon \in\left(0, \frac{1}{2}\right)$ be arbitrary, and let $\phi_{0}$ be a $c^{1,1}$ function on the interval $\left[-\varepsilon^{2}, 1\right]$ with $\phi_{0}\left(-\varepsilon^{2}\right)=\phi_{0}(1)=0$, $\phi_{0}^{\prime \prime}=1$ on $\left[-\varepsilon^{2}, \varepsilon\right],\left|\phi_{0}^{\prime \prime}\right| \leq 2 \varepsilon$ on $[\varepsilon, 1],\left|\phi_{0}^{\prime}\right| \leq 2 \varepsilon$ on $\left[-\varepsilon^{2}, 1\right]$, $t \phi_{0}^{\prime}(t) \leq 2 \phi_{0}(t)$ on $\left[-\varepsilon^{2}, 1\right]$, and $0<\phi_{0}<2 \varepsilon$ on $\left(-\varepsilon^{2}, 1\right.$ ). (It is left to the reader to check that such a function $\phi_{0}$ exists.) Then we let

$$
\phi(t)=K^{-2} \phi_{0}(K t) \quad(K \geq 1 \text { arbitrary })
$$

With such a choice of $\phi$ (and with $k=1$ ) one readily checks that (1.4)-(1.6) imply, for $-\varepsilon^{2} \leq K t \leq 1$, that

$$
M_{0}(\tilde{w}) \geq \mu y_{1}+\alpha y_{2} E(t)-C \alpha K^{-1} y_{2}^{2}\left(1+\varepsilon y_{2}\right)^{2}-L y_{1}^{2}
$$

where $E(t) \geq \frac{1}{2}$ for $-\varepsilon^{2} \leq K t<\varepsilon$ and $E(t) \geq-6 \varepsilon$ for $\varepsilon \leq K t \leq 1$, provided $\alpha|D f| \leq 1$. This last restriction is guaranteed if we take $8 \alpha y_{2}^{2} \varepsilon \leq 1$. We now restrict $\alpha, \varepsilon$ such that

(1.8) $\varepsilon K \leq 1,9 \mu \varepsilon^{2} / K<9 \alpha<\mu^{*} \varepsilon /(K C), \mu^{*}=\min \{\mu, 1\}$.

Notice that $K$ is still arbitrary. Also, we can ensure that $\alpha f \leq \delta$, $\alpha|D f| \leq 1$ for $0 \leq y_{2} \leq K,-\varepsilon^{2} \leq K t \leq 1,\left|y_{1}\right| \leq \delta$, by taking

$$
8 \alpha \varepsilon K \leq \delta
$$

(Then $\delta \in(0,1)$ is also arbitrary.)

Subject to these restrictions one now easily checks from (1.7) the 
following:

(i) for $-\varepsilon^{2} \leq K t \leq \varepsilon$ and $0 \leq y_{2} \leq K$ and $\left|y_{1}\right| \leq \delta$,

$$
\begin{aligned}
M_{0}(\tilde{w}) & \geq \mu y_{1}-L y_{1}^{2}+\alpha y_{2}\left[\frac{1}{2}-4 C \alpha K^{-1} y_{2}^{2}\right] \\
& \geq-2 \frac{\mu \varepsilon^{2}}{K} y_{2}+\frac{\alpha y_{2}}{4} \geq 0 ;
\end{aligned}
$$

(ii) for $\varepsilon \leq K t \leq 1$ and $0 \leq y_{2} \leq K$ and $\left|y_{1}\right| \leq \delta$,

$$
\begin{aligned}
M_{0}(\tilde{w}) & \geq \frac{\mu}{2} y_{1}-6 C \alpha y_{2}\left[\varepsilon+K^{-1} y_{2}\right] \\
& \geq y_{2}\left[\frac{\mu \varepsilon}{2 K}-9 C \alpha\right] \geq 0 .
\end{aligned}
$$

Thus subject to the restrictions (1.8), (1.9) we conclude

(1.10) $M(\tilde{w}) \geq 0$ for $-\varepsilon^{2} \leq K t \leq 1,0<y_{2} \leq k \delta,\left|y_{1}\right| \leq \delta$.

This will be used in the next section in the manner we have already briefly alluded to above.

Next we wish to consider the possibility that, instead of (1.6), there holds

$$
M(\omega) \leq-\left.\left.\mu\right|_{y_{1}}\right|^{k},\left|y_{1}\right| \leq \delta
$$

where $k$ is a positive integer and $\mu>0$.

In this case we take arbitrary $\varepsilon \in\left(0, \frac{1}{2}\right)$ and take any

$\phi \in C^{1,1}([-1,1])$ such that $0<\phi$ on $(-1,1),\left|\phi^{\prime}\right|+|\phi| \leq \varepsilon$ on $[-1,1], \phi(t)=\phi(-t), t \in[-1,1], \phi(-1)=\phi(1)=\phi^{\prime}(-1)=\phi^{\prime}(1)=0$, (1.12) $\phi^{\prime \prime} \leq-\frac{3}{4}$ on $(-\varepsilon, \varepsilon),|\phi \prime| \leq 2 \varepsilon$ on $[-1,1] \sim[-\varepsilon, \varepsilon]$.

Provided that $(k+2) \alpha_{2}^{k+1} \varepsilon \leq 1$ (which ensures that $\alpha|D f| \leq 1$ ), we deduce from $(1.4)$ and (1.11) the following:

(i) for $|t| \leq \varepsilon, 0<y_{2} \leq 1,\left|y_{1}\right| \leq \delta$,

$$
\begin{aligned}
M_{0}(\tilde{w}) & \leq-\mu\left|y_{1}\right|^{k}+\alpha y_{2}^{k}\left[-\frac{1}{4}+4(k+2)^{2} \varepsilon+2 C\left(\left|y_{1}\right|+\alpha\right)\right] \\
& \leq 0
\end{aligned}
$$




$$
\begin{aligned}
& \text { provided } \delta+\alpha \leq(16 C)^{-1}, \varepsilon \leq 1 /\left(32(k+2)^{2}\right) ; \\
& \text { (ii) for } t \in[-1,1] \sim[-\varepsilon, \varepsilon], 0<y_{2} \leq 1,\left|y_{1}\right| \leq \delta, \\
& M_{0}(\tilde{w}) \leq-\mu \varepsilon^{k} y_{2}^{k}+\alpha y_{2}^{k}\left[\varepsilon\left(1+4(k+2)^{2}\right)+2 C\left(\left|y_{1}\right|+\alpha\right)\right] \\
& \leq y_{2}^{k}\left|-\mu \varepsilon^{k}+\alpha\left(1+4(k+2)^{2}\right) \varepsilon+2 C(\alpha+\delta)\right| \\
& \leq 0, \\
& \text { provided } \alpha \leq \mu \varepsilon^{k} /\left(1+4(k+2)^{2}\right), \alpha+\delta \leq \mu \varepsilon^{k /(2 C) .} .
\end{aligned}
$$

Thus in any case we have

(1.13) $M(\tilde{w}) \leq 0$ for $\left|y_{1}\right| \leq \delta, 0<y_{2} \leq \delta, \quad\left|y_{1}\right| / y_{2} \leq 1$,

provided we take $\varepsilon=1 /\left(32(k+2)^{2}\right)$ and provided the constants $\alpha$, $\delta$ are chosen such that

$$
\text { (1.14) } \alpha \leq \mu^{*} \delta \varepsilon^{k} /\left(1+4(k+2)^{2}\right), \delta \leq \mu^{*} \varepsilon^{k} /(16 C), \mu^{*}=\min \{\mu, 1\} .
$$

Notice that subject to these restrictions we automatically have $\alpha|D f| \leq I$ and $\alpha f \leq \delta$.

We should also mention that in each of the above cases we have chosen $\phi$ such that $t \phi^{\prime}(t) \leq 2 \phi(t)$. This ensures that $\partial f / \partial y_{2}>0(f$ as in (1.3)) and hence that the graph $y_{3}=\tilde{w}\left(y_{1}, y_{2}\right)$ does transform to a graph $x_{3}=w\left(x_{1}, x_{2}\right)$ are required.

\section{Main results}

We now use the barrier results of $\delta 1$ to prove the theorems mentioned in the introduction. First we make a precise statement of the theorem concerning continuity of $u$ at points $\xi \in \Sigma$ when $H_{\Sigma}$ satisfies the conditions

$$
H_{\Sigma}(\xi)=0,\left|\partial_{\Sigma} H_{\Sigma}\right| \geq \beta>0 \text { on } \partial \Omega \cap B_{\delta}(\xi), \quad \Sigma \in C^{2,1}
$$

where $\beta, \delta$ are positive constants and where $\partial_{\Sigma}{ }^{H}$ denotes the tangential derivative of $H_{\Sigma}$ along $\Sigma$. 
THEOREM 1. Suppose (2.1) holds, and let $u$ be any $C^{2}(\Omega) \cap W^{1,1}(\Omega)$ solution of the non-parametric least area problem (0.1). Then

$$
|u(x)-\psi(\xi)| \leq c|x-\xi|^{1 / 3},|x-\xi|<\delta, x \in \Omega,
$$

and

$$
|\bar{u}(x)-\psi(\xi)| \leq c|x-\xi|,|x-\xi|<\delta, \quad x \in \Sigma,
$$

where $c$ depends only on $\Sigma, \sup _{B_{\delta} \cap \Omega}|u|$ and Lip $\psi$, and where $\bar{u}$

denotes the trace of $u$ on $\Sigma$.

Proof. We may assume that $\xi=0, \psi(0)=0$, and that $(0,1)$ is the inward pointing normal to $\partial \Omega$ at 0 .

For a suitable portion $\Sigma$ of $\partial \Omega$ the condition (2.1) then guarantees that (1.5) holds for suitable $\mu, \delta$, and hence by the discussion of $\delta 1$ we know that for any preassigned $K \geq 1$ there is a neighbourhood $U$ of $\Sigma \cap\left\{x:\left|x_{1}\right|<\delta\right\}$ with $U \cap \partial \Omega=\Sigma \cap\left\{x:\left|x_{1}\right|<\delta\right\}$ and a

$C^{0}(\overline{U \cap \Omega}) \cap C^{2}(U \cap \Omega)$ function $w$ satisfying $M(w) \leq 0, w(0)=0$, $\left.w\right|_{\partial U \cap \Omega} \geq K \delta$, and $w(x) \geq K\left|x_{1}\right|, x \in \Sigma \cap U$. Thus taking $K$ such that $K \delta \geq 2 \max \{\sup |\psi|, \operatorname{Lip} \psi\}$ we can assert that $w \geq \psi$ on $\sum \cap U$ and $w \geq u$ on $\partial U \cap \Omega$. (Here we assume $\delta$ is chosen small enough to ensure that (1.1) holds with $L \delta<1$. ) Hence by standard barrier results for solutions to $(0.1)$, we conclude that $u \leq w$ everywhere in $U \cap \Omega$. The results (2.2), (2.3) now follow because, by construction of $w$, we have $w(x) \leq c|x|^{1 / 3}$ for $x \in U \cap \Omega$ and $w(x) \leq c|x|$ for $x \in \Sigma \cap U$, with $c$ depending only on $\beta, \delta$, and $K$.

We want to conclude this paper by presenting, for each integer $k \geq 1$, examples to show that $u$ may have no limit at $\xi \in \partial \Omega$ even when $\Sigma=\partial \Omega$ is $c^{k+1,1}$ and when the curvature $H_{\Sigma}(x) \geq-|x|^{k}$, $x \in \Sigma \cap B_{\delta}(\xi)$, for some $\delta>0$. Indeed we give an example to show that $u$ may have no limit at $\xi$ even when the function $\psi$ of $(0.1)$ is constant in a neighbourhood of $\xi$.

To do this we take any two bounded domains $\Omega_{0}$ and $\Omega_{1} \subset \mathbf{R}^{2}$ with $0 \in \partial \Omega_{0} \cap \partial \Omega_{1}, \quad \Sigma_{j}=\partial \Omega_{j} \cap B_{2 \delta}(0)$ connected, $\Omega_{0} \subset \Omega_{1}$, 
$\Omega_{1} \sim \Omega_{0} \subset B_{\delta / 2}(0)$, and such that the following conditions hold, in which $H_{j}$ denotes the curvature of $\Sigma_{j}$ relative to the inward unit normal $n_{j}$ of $\Sigma_{j}:$

$$
\begin{gathered}
\left\{\begin{array}{r}
H_{j} \leq 0 \text { on } \Sigma_{j}, \quad \eta_{j}(0)=(0,1), \\
\left|n_{j}(x)-n_{j}(0)\right|<\frac{1}{2}, x \in \Sigma_{j}, j=0,1 ;
\end{array}\right. \\
-\left|x_{1}\right|^{k} \leq H_{0}(x) \leq-\frac{3}{2}\left|x_{1}\right|^{k}, x \in \Sigma_{0},\left|x_{1}\right|<\delta ;
\end{gathered}
$$

and

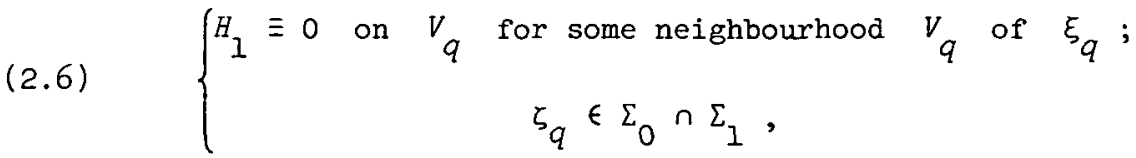

where $\left\{\xi_{q}\right\},\left\{\zeta_{q}\right\}$ are sequences of points in $\mathbb{R}^{2}$ with

$\lim \xi_{q}=\lim \zeta_{q}=0$. (It is left to the reader to check that such domains $\Omega_{0}$ and $\Omega_{\perp}$ can be constructed in such a way that both $\partial \Omega_{0}$ and $\partial \Omega_{1}$ are $c^{k+1,1}$. We emphasize that, apart from the above restriction, $\Omega_{0}$ and $\Omega_{1}$ are quite arbitrary.)

Now let $U$ be an arbitrary neighbourhood of $\left\{x \in \Sigma_{0}:\left|x_{1}\right|<\delta\right\}$ with $U \cap \partial \Omega_{0}=U \cap \Sigma_{0}=\left\{x \in \partial \Omega_{0}:\left|x_{1}\right|<\delta\right\}$. Evidently, since $H_{0}<0$ on $U \cap\left(\Sigma_{0} \sim\{0\}\right)$, we can find a solution $u_{0}$ of the problems (0.1) with $\Omega_{0}$ in place of $\Omega$ and $\psi_{0}$ in place of $\psi$, where $\psi_{0} \equiv 1$ on $\Sigma_{0} \cap\left\{x:\left|x_{1}\right|<\delta / 2\right\}$ and

$$
\begin{aligned}
& \lim _{\bar{\zeta} \rightarrow x} u_{0}(\xi)<-1 \text { for all } x \in \partial U \cap \bar{\Omega}_{0} \text {. } \\
& \xi \in U \cap \Omega_{0}
\end{aligned}
$$

(One achieves this by taking $\psi_{0} \leq 1$ everywhere on $\partial \Omega_{0}$ and $\psi_{0} \leq-L$ on $\partial \Omega_{0} \sim\left\{x:\left|x_{1}\right|>3 \delta / 4\right\}$, where $L$ is a sufficiently large constant. $\}$

We now introduce coordinates $y_{1}, y_{2}, y_{3}$ for $\mathrm{R}^{3}$ as described in $\S 1$. 
Then (2.5) implies that (1.11) holds with $\Sigma_{0}$ in place of $\Sigma$ and with $\mu=\frac{3}{2}$. Hence, by the discussion of the latter part of $\$ 1$, we can find a function $\tilde{w}=\tilde{w}\left(y_{1}, y_{2}\right)=\omega+\alpha y_{2}^{k+2} \phi\left(y_{1} / y_{2}\right)$ with $M(\tilde{w}) \leq 0$ on $W=\left\{\left(y_{1}, y_{2}\right): 0<y_{2}<\delta,\left|y_{1}\right| / y_{2}<1,\left|y_{1}\right|<\delta\right\}$ and with graph $\tilde{w}$ corresponding, in the $\left(x_{1}, x_{2}, x_{3}\right)$ coordinates, to graph $w$, where $w \in C^{2}\left(U \cap \Omega_{0}\right)$ with $U \cap \partial \Omega_{0}=\left\{x \in \Sigma_{0}:\left|x_{1}\right|<\delta\right\}$. From construction $w$ has the properties:

$\frac{\partial w}{\partial \eta_{0}}=\infty \quad$ (in the sense that $\lim _{\substack{\xi \rightarrow x \\ \xi \in U \cap \Omega_{0}}} D w(\xi) \cdot \eta_{0}(x)=\infty$ for each $x \in \Sigma_{0} \cap U$ ), $0<w \leq \delta$ on $U \cap \Omega_{0}, \quad w=\delta$ on $\partial U \cap \Omega_{0}$, $M(\omega) \geq 0$ on $U$.

Hence we have $M(-w) \leq 0, \partial(-w) / \partial \eta_{0}=-\infty,-\delta \leq-w<0$ on $U \cap \Omega_{0}$, $-w=-\delta$ on $\partial U \cap \Omega_{0}$. Since, as described above, we can arrange $u_{0}<-1<-w$ on $\partial U \cap \Omega_{0}$, we then deduce from a standard comparison principle that $u_{0} \leq-w$ on $\bar{U}$. In particular we deduce $u_{0} \leq 0$ on $U \cap \partial \Omega_{0}$.

If we now let $u_{1}$ be the solution of (0.1) with $\Omega_{1}$ in place of $\Omega$ and with $\psi_{1}$ in place of $\psi$, where $\psi_{1} \leq 1$ everywhere and $\psi_{1}=\psi_{0}$ on $\partial \Omega_{0} \cap \partial \Omega_{1}$. Then, since by the maximum principle $u_{1} \leq 1 \leq \psi_{0}$ on $\Sigma_{0} \cap \Omega_{1}$, we deduce $u_{1} \leq u_{0}$ on $\Omega_{0}$. On the other hand it is standard, since $H_{1} \equiv 0$ on $V_{q}$, that $u_{1} \equiv \psi_{1}$ on $V_{q}$. Combining these facts we have, for all $q$ large enough to ensure that $\left|\xi_{q}\right|<\delta / 2$ and $\left|\zeta_{q}\right|<\delta / 2, u_{1}\left(\xi_{q}\right)=1$ and $u_{1}\left(\zeta_{q}\right) \leq u_{0}\left(\zeta_{q}\right) \leq 0$. Thus $u_{1}$ is not continuous at 0 .

Thus we have established the following theorem.

THEOREM 2. Let $\Omega_{0}, \Omega_{1}$ be any $c^{k+1,1}$ domains as described above 
(in (2.4), (2.5), (2.6)) and let $u_{1}$ be the solution of (0.1) with $\Omega_{1}$ in place of $\Omega$ and with $\psi_{1}$ in place of $\psi .\left(\psi_{1}\right.$ as described above. $)$

Then $\lim _{x \rightarrow 0} u_{1}(x)$ does not exist. In fact the trace of $u_{1}$ on $2 \Omega_{1}$ has $x \in \Omega_{1}$

no limit at $x=0$.

\section{References}

[1] Enrico Giusti, "Superfici cartesiane di area minima", Rend. Sem. Mat. Fis. Mizano 40 (1970), 135-153.

[2] Enrico Giusti, "Boundary behavior of non-parametric minimal surfaces", Indiana Univ. Math. J. 22 (1972), 435-444.

[3] J. Serrin, "The problem of Dirichlet for quasilinear elliptic differential equations with many independent variables", Philos. Trans. Roy. Soc. London Ser. A 264 (1969), 413-496.

[4] Leon Simon, "Boundary regularity for solutions of the non-parametric least area problem", Ann of Math. (2) 103 (1976), 429-455.

[5] Leon Simon, "Global estimates of Hölder continuity for a class of divergence-form elliptic equations", Arch. Rational Mech. Anal. $56(1974), 253-272$.

[6] Neil S. Trudinger, "The boundary gradient estimate for quasilinear elliptic and parabolic differential equations", Indiana Univ. Math. J. 21 (1972), 657-670.

Department of Mathematics, Institute of Advanced Studies, Australian National University, PO Box 4, Canberra, ACT 2600, Australia. 